\title{
« Frankreich für Historiker »
}

\section{Marion Deschamp, Guillaume Garner et Juliette Guilbaud}

\section{OpenEdition}

\section{Journals}

Édition électronique

URL : http://journals.openedition.org/ifha/122

DOI : $10.4000 /$ ifha. 122

ISSN : 2198-8943

\section{Éditeur}

IFRA - Institut franco-allemand (sciences historiques et sociales)

\section{Édition imprimée}

Date de publication : 6 février 2011

Pagination : $32-37$

ISSN : 2190-0078

\section{Référence électronique}

Marion Deschamp, Guillaume Garner et Juliette Guilbaud « « Frankreich für Historiker » », Revue de I'IFHA [En ligne], 3 | 2011, mis en ligne le 01 octobre 2012, consulté le 03 mai 2019. URL : http:// journals.openedition.org/ifha/122 ; DOI : 10.4000/ifha.122

Ce document a été généré automatiquement le 3 mai 2019.

(CIFHA 


\title{
« Frankreich für Historiker »
}

\author{
Marion Deschamp, Guillaume Garner et Juliette Guilbaud
}

\section{NOTE DE L'ÉDITEUR}

Journées d'études, Francfort-sur-le-Main, 25-26 novembre 2010

1 Les 25 et 26 novembre 2010 se sont tenues, à l'initiative de Thomas LIENHARD (IFHA), trois demi-journées de rencontre consacrées à la présentation du paysage historique français à l'usage des historiens allemands, jeunes ou confirmés. Le but de cette manifestation était d'offrir une information groupée et (donc) efficace sur trois grands thèmes: les institutions de recherche et d'enseignement supérieur, l'accès à la documentation et un aperçu de la situation actuelle de l'historiographie française et des débats la traversant.

Le premier volet de cette rencontre fut d'abord consacré à la présentation de quelques institutions d'enseignement supérieur et de recherche. Après que Marc SMITH, Igor MOULLIER et Brigitte MAZON eurent présenté ces trois institutions si typiquement et étrangement françaises que sont, pour nombre d'étudiants allemands, l'École nationale des chartes, les Écoles normales supérieures et l'École des hautes études en sciences sociales, Thomas LIENHARD (IFHA) aborda un terrain plus familier en évoquant l'université Paris I, avant que Mareike KÖNIG close cette séquence par une intervention consacrée à l'Institut historique allemand de Paris. La seconde moitié de l'après-midi prit un tour plus concret avec deux mises au point sur les systèmes d'échange permettant d'étudier et de faire de la recherche en France, présentées par Marjorie BERTHOMIER (univ. Paris V) et Klaudia KNABEL (DAAD).

En raison notamment d'une histoire fortement marquée par la centralisation, la France a une organisation des archives et des bibliothèques très différente de la situation allemande, comme le souligna Igor MOULLIER dans sa présentation des archives, des bibliothèques et des ressources disponibles sur l'internet. Une autre caractéristique française fut également abordée lors de cette matinée par Valérie HANIN (L'Histoire) : l'importance des canaux de diffusion des acquis de la recherche historique en direction 
du grand public, à travers la revue L'Histoire et les Rendez-vous de l'histoire de Blois même si ces derniers ont depuis deux ans leur pendant à Weimar.

Plat de résistance et dessert de la rencontre, la troisième demi-journée proposa six interventions sur le paysage historiographique français actuel.

Dans son intervention, François DosSE (univ. Paris XII) décrivit le tournant historiographique opéré, selon lui, par les chercheurs français depuis les années 1980, comme une "histoire élevée au second degré». Partant du constat d'une crise de l'historicité, liée à l'opacification de l'avenir (la fin annoncée de l'histoire n'ayant pas eu lieu) et caractérisée par la remise en cause de toute forme de téléologie historique, les historiens de la postmodernité auraient opté pour une pratique plus réflexive de l'histoire. Le tournant critique des années 1988-1989, remettant en cause certains dogmes hérités de l'école des Annales, joua notamment un rôle majeur dans la reconfiguration des champs d'investigation de la discipline. F. Dosse présenta ainsi les acquis de l'histoire mémorielle - propulsée sur le devant de la scène historienne par les Lieux de mémoire de Pierre Nora et irriguée par les réflexions de Paul Ricœur-comme un exemple paradigmatique de cette écriture d'une histoire au second degré, proposant des questionnements nouveaux sur les relations entre l'histoire et la mémoire, et l'omniprésence du passé dans nos sociétés actuelles. F. DOSSE s'arrêta ensuite sur le retour en grâce de deux «formats » d'histoire, longtemps relégués dans les basses-fosses de la discipline au nom d'une histoire totale, privilégiant le général sur le particulier: la biographie et l'événement. L'explosion biographique, tout comme la renaissance de l'événement, participent bien, selon F. DOSSE, d'une réhabilitation des phénomènes singuliers, non pas seulement étudiés au titre de leur exemplarité supposée, mais aussi au nom de la richesse des trajectoires particulières et d'une certaine irréductibilité du sens au traitement sériel et statistique. Enfin, F. DossE insista sur une tendance profonde de l'historiographie actuelle, qui est de considérer la situation médiane de l'histoire, produit bâtard de la science et de la fiction - comme l'enseignait Michel de Certeau -, pour en dévoiler toutes les potentialités interprétatives et scripturales.

C'est au champ en pleine expansion des études postcoloniales que Pascale BARTHÉLÉMY (ENS Lyon) consacra son intervention («Phénomène médiatique ou champ de recherches ? Les études postcoloniales en France »). Elle rappela dans un premier temps que l'année 2005 fut décisive dans le processus d'émergence du postcolonial en France : le vote de la loi du 23 février "portant reconnaissance de la Nation et contribution nationale en faveur des Français rapatriés», l'«Appel pour les assises de l'anticolonialisme postcolonial » lancé par le collectif des Indigènes de la République, et enfin la parution de l'ouvrage La Fracture coloniale. La société française au prisme de l'héritage colonial, furent autant d'événements politiques et scientifiques qui stimulèrent, autour de la portée du passé colonial de la France, un débat qui était entré depuis quelques années dans une phase d'incubation. Les controverses autour des études postcoloniales ne sont, aujourd'hui encore, pas éteintes. Ces études sont en effet l'objet de critiques parfois vigoureuses, qui leur reprochent d'une part - sur un plan scientifique - le flou et l'emploi d'un terme monolithique, conduisant à une essentialisation de phénomènes complexes ; d'autre part - sur un plan politique -, la fragilisation de la cohésion sociale dont elles seraient porteuses. En dépit de ces critiques, les études postcoloniales ont porté un renouvellement des objets et des méthodes qui ne peut être nié, mais qui est en partie brouillé par le caractère souvent passionnel des débats suscités par ce courant. 
7 Il revenait à Jean-François BERT (Institut interdisciplinaire d'anthropologie du contemporain, UMR 8177, EHESS-CNRS, Paris) la lourde tâche d'expliquer la postérité de Michel Foucault, en particulier chez les historiens dont Foucault chercha par ailleurs toujours à se démarquer. Un certain nombre de lieux communs restent attachés au philosophe - ses thèmes d'étude, autrefois jugés marginaux, ses méthodes, son style, etc. La réception de Foucault est toujours extrêmement difficile à mesurer, car la référence à ses travaux n'est pas toujours explicite ni assumée, mais plus souvent "souterraine ». Outre ses excès théoriques, Foucault reste une lecture stimulante pour l'historien : elle l'invite à dépasser les cadres disciplinaires et l'oblige à se remettre en question. Et J.-F. BERT de passer en revue un certain nombre des travaux de Foucault, pour montrer en quoi ils se démarquent d'une vision jugée «trop classique " de l'historiographie. Pour finir, l'orateur revint sur quatre exemples de ce qu'il appelle les «usages historiens » de Foucault: les travaux de Paul Veyne, ceux de Roger Chartier; et plus récemment les ouvrages de deux philosophes, celui de Grégoire Chamayou sur l'expérimentation sur les êtres humains aux XVIIIe-XIXe siècles, et celui d'Elsa Dorlin sur la «nosopolitique » (la construction et instrumentalisation des catégories sain/malsain, en particulier pendant la période coloniale).

Blaise WILFERT (ENS Paris) aborda les rapports de Pierre Bourdieu avec la profession historienne et l'enquête historique («Bourdieu et les historiens »), en rappelant d'abord que Bourdieu souligna de manière récurrente l'historicité de toutes les sciences sociales et le rôle fondamental de l'historicisation non seulement des objets de l'enquête des sciences sociales, mais aussi et surtout de la position de l'enquêteur et des outils d'analyse qu'il mobilise. Cependant, l'accueil des travaux de Bourdieu chez les historiens fut mitigé pour trois raisons principales: l'hostilité d'une partie des historiens à la sociologie, depuis la querelle entre Simiand et les méthodistes; la conjoncture particulière des années 1970-2000, marquées par le déclin de l'école des Annales et la réhabilitation du «sujet»; enfin, les réactions aux prises de position publiques de Bourdieu dans les années 1990. B. WILFERT rappela cependant que des concepts, des configurations de concepts ou des œuvres entières de Bourdieu ont été l'objet d'une réception et d'une appropriation actives de la part de certains historiens français: deux exemples marquants en sont l'application de la notion de "champ » en histoire de l'activité littéraire comme en histoire politique, et la portée de la sociologie bourdieusienne du discours, comme lutte pour l'imposition de schèmes de classement, dans l'histoire des élites sociales comme dans l'histoire des sciences humaines.

Emmanuelle LOYER (IEP Paris), quant à elle, tint à rappeler le parcours chaotique suivi par l'histoire politique au sein de l'historiographie française d'après-guerre. Délégitimée d'abord par le structuralisme puis, à partir des années 1970, par un braudélisme dominant dans le pré carré français, l'histoire politique a su, selon E. LOYER, se défaire du reproche de n'être que l'écume événementielle du temps historique, en adoptant les méthodes de l'anthropologie historique et de l'histoire des mentalités, pour devenir une «histoire culturelle du politique ", reconquérant peu à peu ses quartiers de noblesse. D'abord circonscrite aux temps contemporains et léguée en apanage à quelques institutions, comme l'Institut d'études politiques dominé par le magistère de René Rémond, elle bénéficia au cours des années 1980 de plusieurs travaux d'importance qui contribuèrent à sa notoriété retrouvée: l'ouvrage de Robert Paxton sur la France de Vichy, bientôt complété par les travaux de François Bédarida et Jean-Pierre Azéma, marquèrent bien le réveil de l'intérêt médiatique et public, avant de devenir académique, 
pour ce nouveau type d'histoire. Dans les années 1980, l'histoire culturelle s'annexait ouvertement au terrain politique, l'irriguant de ses interrogations. Et E. LOYER de développer trois exemples de ces nouveaux objets. En évoquant d'abord les études nourries d'anthropologie historique qui s'emploient à décrypter la fabrication du politique à travers et dans ses objets visibles: rites, iconographies, représentations symboliques, etc. En abordant ensuite la constitution d'un objet très français, celui des politiques culturelles, d'ailleurs coïnvesti par les historiens et les sociologues, qui s'emploient à inventer ensemble une "sociohistoire » des politiques publiques. Enfin, en se penchant sur une historiographie récente, qui cherche à tirer les fils de la mémoire dans la surgie d'un événement particulier, c'est-à-dire à retrouver les dynamiques politiques des usages de la mémoire. E. Loyer conclut alors sur le double mouvement opéré par l'histoire culturelle du politique, faisant sortir le politique de la politique, pour repolitiser un certain nombre de lieux sociaux, artistiques ou intellectuels.

Pour clore cet après-midi, Nicolas VERDIER (CNRS, Paris) se pencha sur les tensions disciplinaires et pratiques transdisciplinaires entre géographie et histoire, à travers la notion d'espace. Dans une première partie, chronologique, l'orateur rappela les conditions d'autonomisation de la discipline géographique dans le champ académique et universitaire, et ses évolutions au cours du siècle dernier. Longtemps considérée comme une sous-discipline de l'histoire, la géographie s'émancipa de cette « tutelle » au début du XXe siècle, sous l'impulsion de Paul Vidal de la Blache qui transforma sa chaire de géographie historique en chaire de géographie. Cette remise en cause de la suprématie de l'histoire n'allait cependant pas de soi, comme le montrèrent les multiples hésitations, réorientations et développements de la géographie au siècle dernier. Les années 1940 virent cohabiter non sans friction plusieurs approches de la notion d'espace : géographie historique (Le Bras, Dainville), géographie humaine rétrospective (Dion) ou encore géohistoire (Braudel). Les nombreuses et désormais fameuses thèses d'histoire régionale des années 1950-1970 (celles de Duby, Le Roy-Ladurie, Goubert, etc.) ne purent quant à elles échapper à la critique, accusées d'être des histoires dans l'espace plutôt que de l'espace (Perrot). Dans les années 1980, la discipline géographique s'est sans conteste renouvelée, à la faveur de travaux d'historiens réinterrogeant la notion d'espace, à partir de concepts comme ceux de territoire et de mémoire. À la suite de cette chronologie, $\mathrm{N}$. Verdier revint sur les choix méthodologiques et thématiques de ces vingt dernières années, à travers lesquels se rejoue sans cesse la tension entre histoire et espace. Parmi les domaines les plus dynamiques furent évoquées la géographie historique (encore largement marquée par les points de vue disciplinaires tantôt des historiens, tantôt des géographes), la géohistoire associée à l'utilisation des chorèmes, la modélisation spatiotemporelle, l'archéohistoire et l'archéologie spatiale, et enfin l'histoire du territoire. Les thèmes de recherche privilégiés sont le paysage, les circonscriptions et les réseaux. Si le manque d'homogénéité dans l'utilisation des concepts d'espace et de territoire reste problématique, l'intérêt croissant des historiens pour ces deux notions dans le cadre de leurs travaux ne peut que réjouir le géographe. 